\title{
Does the professional and working context of United Kingdom clinicians predict if they use practices to support patients with long term conditions to self manage?
}

Joanna Kosmala-Anderson, Louise M. Wallace, Andrew Turner

Applied Research Centre in Health and Lifestyle Interventions, Coventry University, Coventry, United Kingdom

Submitted: 14 September 2009

Accepted: 16 October 2009

Arch Med Sci 2010; 6, 5: 815-821

DOI: 10.5114/aoms.2010.17100

Copyright $\odot 2010$ Termedia \& Banach

\begin{abstract}
Introduction: Our study examines how the professional and employment context may influence clinicians' practice self management support for patients with long term conditions (LTC).

Material and methods: We surveyed clinicians working with patients with depression, chronic obstructive pulmonary disorder (COPD), chronic musculo skeletal pain and diabetes.

Results: Clinicians most frequently endorsed items on a scale concerned with patient centeredness, and less frequently endorsed items concerned with clinical and organizational self management support. The most important factors predicting these latter activities were the intensity of working experience with patients with LTC and attending professional training addressing the principles and practice of self management support. Practicing patient centeredness was endorsed by nearly all respondents, and so was not sensitive to variation on work variables.

Conclusions: The interaction of training and intensity of work with patients with LTC seems to have the most powerful effect on undertaking clinical and organizational self management support practices. To facilitate clinicians' practice of self management support for patients with LTC it is very important to provide relevant professional training and to build specialized patient care teams with professionals having complimentary skills.
\end{abstract}

Key words: long term conditions, self management, professional training.

\section{Introduction}

Chronic conditions are increasingly the main concern of the healthcare systems throughout the world [1]. In the United Kingdom seventeen and a half million people report having a long term condition (LTC) and this number is constantly increasing [2]. Healthcare systems must change in response to increasing demand to provide optimal, continuous care for patients living with a LTC, in which patients themselves have a key role to play. The Chronic Care Model (CCM) has been developed in the USA to introduce system changes, developing prepared proactive healthcare teams able to engage in productive interactions with informed and activated patients [1]. These productive interactions assume clinicians and patients will work in partnership, bringing complimentary skills and knowledge to their relationship [3]. This means moving away from traditional healthcare
Corresponding author: Joanna Kosmala-Anderson PhD Applied Research Centre for Health and Lifestyle Interventions Coventry University Priory Street Coventry CV1 5FB, United Kingdom Phone: +2476887189 Fax: +24 76795987 E-mail: j.kosmalaanderson@coventry.ac.uk 
models where the clinicians are expected to manage their patients' conditions towards a model where patients takes responsibility for their health and, using the expertise and support offered by clinicians, engage in self management. Achieving this requires not only a significant cultural shift and developing patients' readiness to fully participate in their care, but also changing clinicians' beliefs about their role in promoting self management, followed by acquiring new practice behaviours and skills, and new ways of organizing services to support these new practices.

While there is considerable research on the application of behaviour change theories to health behaviour changes [4-7], little is known on how to enable clinicians to apply these models and techniques within everyday clinical practice. This is in part because there is no consensus on the style of consultation and the specific behavioural skills required by the clinician in relation to their role in the patients' pathway of care. Some studies suggest that developing a trusting clinician-patient relationship is a key factor [8-12], but such studies do not specify how this is to be manifested at a behavioural level. Others go some way to breaking down this concept into objectives. For example, it has been shown that clinicians' ability to identify and adequately respond to patients' ideas and emotions regarding their illness, reaching common ground about the illness and its treatment, as well as agreeing the roles that the patient and clinician will assume can significantly enhance the medical encounter and prepare both parties for further work on developing a common self management plan [13]. It has also been shown that before patients can properly engage in self management they need to obtain sufficient, clear information about their condition as well as services available [14]. Newman [15] has recommended that staff cultivate an "ethos of self-management and self-efficacy" among their patients which would decrease dependency. Clinicians also have to learn how to apply behaviour change techniques to develop and foster self-management skills among their patients [14].

Reviewing the research on how to measure self management support and health promotion practices relevant to clinicians working with people with a LTC, we found there were several key practices found to be effective but no one measurement scale relevant to the UK context. As described in Kosmala-Anderson et al. (in press) we developed a measure that captures these practices and encompass the style of the consultation, the behavior change practices and practices that link the patient into a supportive system of healthcare and community resources. This study seeks to ascertain the frequency with which such practices are used, and by whom, in order to better target service quality improvement, personal development and training resources, to enable all practitioners in the pathway of care to use their expertise in patient care and in service design optimally to influence the self management of each patient with a LTC. It is possible that practices to support self management will vary according to the clinician's place in the care pathway. Traditionally in the UK, primary care generalist clinicians and secondary care based specialists worked in separate systems with limited communication. Recently more attention is being paid to establishing optimal roles and working relationships of primary care clinicians, specialist and professionals allied to medicine in providing self management support to patients with LTC, and ensuring optimal health outcomes [16-18]. In the UK national health service support for people with a LTC relies upon primary care teams (including community nurses and general practices) that coordinate patient care and support self care [18]. The role of specialists includes establishing the course of treatment, providing expertise to care team and participating in supporting self management. We therefore explore the impact of care sector and of professional role on the range of self management support practices employed. We also explore if factors that managers might use to target training and quality improvement resources are in fact associated with having a wide range of self management support practices. We examine the duration and intensity of current work with people with a LTC by clinicians and clinicians' experience of relevant training, on the range of practices employed.

We tested the following hypotheses:

Hypothesis 1: Clinicians who spend more time on direct care/contact with patients with LTCs are more likely to practice self management support.

Hypothesis 2: There is a positive correlation between the length of experience with patients with LTCS and undertaking self management support practices.

Hypothesis 3: Clinicians who have recently attended training addressing the principles and practice of self management support are more likely to practice self management support.

Hypothesis 4: There is a difference between primary and secondary care clinicians in undertaking self management support practices.

Hypothesis 5: There is a difference between professional groups in engaging in different areas of self management support practices.

Hypothesis 6: There is a difference between clinicians with patient care duties and those in largely managerial or academic roles in practicing organizational self management support. 
To find out more about the relationship between work experience and practices of self management support we employed factorial ANOVA and multiple regression. We tested the contribution of intensity of experience with patients with LTC, attending professional training addressing the principles and practice of self management support and profession, and care type in predicting undertaking Clinical Self Management Support, Patient Centeredness and Organizational Self Management Support.

\section{Material and methods}

\section{Design}

This research was conducted a part of evaluation of a national multi site UK quality improvement programme - Co-Creating Health Initiative $(\mathrm{CCH})$ (http://www.health.org.uk). We invited clinicians who worked with patients with depression, chronic obstructive pulmonary disorder (COPD), chronic musculo skeletal pain and diabetes to complete a web based questionnaire. Data were collected between October 2008 and March 2009.

\section{Sample}

The inclusion criteria was that the clinicians were working in a clinical, academic or clinical support role and spending at least some of their working time in direct care or contact with patients with diabetes, COPD, depression or musculo skeletal pain.

\section{Measures}

The first section of the survey included items describing their age, gender, ethnicity, professional role and employment, the second covered practices in self management support.

To assess the use of self management support practices in clinical consultations for patients with long term conditions we applied the Practices in Self Management Support questionnaire (PSMS) developed by researchers from Coventry University Applied Research Centre for Health and Lifestyle Interventions (Kosmala-Anderson, Wallace, Turner, 2010, in press). The PSMS is a self report measure and comprises three subscales: Clinical Self Management Support (14 items), Patient Centeredness (4 items) and Organization of Services to Support Self Management (7 items). All subscales have very good internal reliability (Cronbach's Alphas: 0.94, 0.78 and 0.85 and all item-total correlations above 0.50). Respondents are asked to rate each statement on a seven point Likert scale in relation to how much they apply each in their current clinical practice.

\section{Procedure}

Participants were invited to complete an online or paper questionnaire. Data were analyzed using SPSS. Where possible, missing data were replaced with means, or if whole sections of survey were skipped they were deleted case wise.

\section{Results}

\section{Characteristic of the sample}

The invitation to complete a web based survey was sent to 1651 clinicians from eight CCH demonstration sites. Up to March 2009 we received 213 surveys fully completed surveys.

The majority of respondents were females (75\%; $n=161)$, mean age 42.7 (SD = 8.9). Most described their ethnic origin as British White $(71.4 \% ; n=152)$. Almost $70 \%$ of respondents $(69.9 \% ; n=149)$ were working in clinical roles and almost all (93.9\%; $n=200$ ) were qualified healthcare practitioners who had been practicing for on average 18 years $(S D=9.4)$. Participants were almost evenly spread between primary and secondary care organizations (32.4\%; $n=69$ and $33.3 \% ; n=71$ respectively; $6.6 \% ; n=14$ were employed in both primary and secondary care organizations and $27.7 \% ; n=59$ did not respond to this question). The majority of respondents were nurses $(35.2 \% ; n=75)$, followed by doctors $(23.9 \% ; n=51)$, professionals allied to medicine $(15.5 \% ; n=33)$ and a very small group of psychologists/counselors (5.8\%; $n=12)$. Almost $20 \%$ of respondents did not state their profession $(19.5 \% ; n=42)$. Most $(67.1 \% ; n=143)$ spent more than $50 \%$ of their working week in direct care/contact with patients with LTCS and has been working with this group of patients for on average 13.8 years $(S D=8.5)$. Over $70 \%$ of respondents $(70.4 \% ; n=150)$ have ever attended professional training addressing the principles and practice of self management support, with most $(67.3 \%$; $n=101)$ within last 2 years, the remaining $32.7 \%$ $(n=49)$ over 2 years ago.

\section{Practices of self management support for patients with long term conditions}

We assessed the frequency of undertaking self management practices for patients with LTC in three different areas: Clinical Self Management Support, Patient Centeredness and Organization of Services to Support Self Management.

The Clinical Self Management Support activity undertaken by the most clinicians was establishing and maintaining a trusting relationship with the patient ( $93 \% ; n=198)$, followed by ensuring that the patient is comfortable with a jointly agreed management plan $(83.6 \% ; n=178)$. Clinicians were least likely to report negotiating an agenda for the 
consultation (42.7\%; $n=91)$ and discussing with patients about joining condition related support groups (37.1\%; $n=79)$. The mean scale score for Clinical Self Management Support was $4.9(S D=1.4)$.

Clinicians most frequently endorsed items on a scale concerned with Patient Centeredness, which includes undertaking self management support activities associated with tailoring services to patients needs and taking an individual approach to their care. Over $80 \%$ of respondents reported customizing treatment recommendations in response to patient's preferences and beliefs $(83.1 \% ; n=177)$ and giving patients individually tailored explanation of their symptoms $(82.2 \% ; n=175)$. The mean score on this subscale was $5.3(\mathrm{SD}=1.6)$.

Clinicians scored the lowest on the Organization of Services to Support Self Management scale, the mean score was $3.6(S D=1.5)$. While just over a half of the clinicians reported jointly agreeing how a patient's progress will be followed up (56.8\%; $n=121$ ), most items were endorsed by less than half of the sample, and around $10 \%$ of all clinicians responded that the activities listed in the subscale do not apply to their practice. Nearly a half of respondents (49.3\%; $n=105)$ engaged in building their patient care team with other professionals, only about a quarter $(26.3 \% ; n=56)$ routinely included a self management record in the patient's documentation and even fewer $(24.4 \% ; n=52)$ gave patients a choice about the care team member who will coordinate their care plan.

\section{Testing hypotheses}

Hypothesis 1: Clinicians who spend more time on direct care/contact with patients with LTCs are more likely to practice self management support.

Our hypothesis was confirmed in relation to two out of three areas of self management support: Clinical Self Management Support $(t=3.1, d f=211$, $p<0.01)$ and Organizational Self Management Support ( $t=2.1, d f=210, p<0.01$ ). Clinicians who spend over a half of their working week with patients with LTCs were significantly more likely to undertake Clinical and Organizational Self Management Support but there was no difference for patient centered practices ( $t=1.8, d f=21$, NS).

Hypothesis 2: There is a positive correlation between the length of experience with patients with LTCs and undertaking self management support practices.

The hypothesis was not supported for Clinical Self Management Support ( $r=0.04$, NS), Patient Centeredness ( $r=0.07$, NS) and Organizational Self Management Support ( $r=0.12$, NS).

Hypothesis 3: Clinicians who have recently attended training addressing the principles and practice of self management support are more likely to practice self management support.
The hypothesis was supported in relation to Clinical Self Management Support $(t=4.3, d f=209$, $p<0.001$ ), and Organizational Self Management Support ( $t=3.1, d f=208, p<0.001$ ), but there was no effect for Patient Centeredness practices $(t=1.3, d f=209, \mathrm{NS})$.

Hypothesis 4: There is a difference between primary and secondary care clinicians in undertaking self management support practices.

Our assumption was not confirmed. There was no difference between primary and secondary care clinicians in undertaking self management support practices in any of the three areas.

Hypothesis 5: There is a difference between professional groups in engaging in different areas of self management support practices.

Our assumption was only confirmed in relation to Organizational Self Management Support $(F=7.9, d f=156, p<0.001)$ but not Clinical Self Management Support and Patient Centeredness. Post hoc tests showed statistically significant differences in undertaking Organizational Self Management Support between doctors and professional allied to medicine ( $p=0.02, m=3.52$ and $m=2.74$ respectively) and nurses and professional allied to medicine $(p<0.001, m=3.89$ and $m=2.74$ respectively) but not doctors and nurses. Both doctors and nurses were significantly more likely to engage in Organizational Self Management Support in comparison to professions allied to medicine.

Hypothesis 6: There is a difference between clinicians with patient care duties and those in largely managerial or academic roles in practicing Organizational Self Management Support.

Clinicians with few patient care duties were significantly more likely to engage in Organizational Self Management Support in comparison to those with greater clinical involvement $(t=-2.3, d f=209$, $p=0.02)$.

\section{Factorial ANOVA and multiple regression}

To find out more about the relationship between work variables and practices in self management support we conducted additional analyses using factorial ANOVA and multiple regression.

First, using factorial ANOVA, we tested the combined effects of care type (primary vs. secondary care) and profession (doctors vs. nurses vs. professionals allied to medicine), and having attended professional training addressing principles and practice of self management support in the past two years (yes vs. no) and the intensity of experience with patients with LTC (less than 50\% vs. more than $50 \%$ of working week) on the practice of self management support sub scale total scores. In regard to Clinical Self Management Support, profession and training effects were statistically 
significant $(F=4.58, p=0.03$ and $F=6.77, p=0.01$ respectively), care type, intensity of working experience with patients with LTCS and interactions between factors were not significant. In relation to Patient Centeredness none of the effects was statistically significant. In regard to Organizational Self Management Support, the effect for profession was statistically significant $(F=13.66, p<0.001)$, as well as the combined effect of intensity of experience with patients with LTC and attending professional training in self management support $(F=4.89, p=0.03)$.

Based on the results of the factorial ANOVA we chose predicators for multiple regression analysis. We tested the contribution of, intensity of experience with patients with LTCs, and attending professional training in self management support in the past two years in predicting undertaking self management support in three sub scale scores. Although we did not find a relationship between care type and practicing self management support we also included this variable to see whether, combined with other factors, it predicts practicing self management support. About $20 \%$ of respondent did not state their profession or care type and missing data were deleted case wise, multiple regression analysis were conducted on 122 eligible cases.

The most important variables predicting undertaking Clinical Self Management Support are intensity of experience with LTCS ( $\beta$ coefficient = $0.20, p<0.01)$ and attending professional training in self management support ( $\beta$ coefficient $=0.31$, $p<0.001)$.

Practicing Patient Centeredness was not predicted by any of variables we chose.

Two of four variables included in the analysis were good predictors of undertaking Organizational Self Management Support, with attending professional training addressing in self management support being the strongest $(\beta$ coefficient $=0.27$, $p<0.001)$, followed by intensity of experience with patients with LTCs $(\beta$ coefficient $=0.20, p=0.02$ ).

\section{Discussion}

Our study examined how the professional and employment context may influence clinicians' practice of self management support for patients with long term conditions (LTCS). We tested the contribution of profession, care type, intensity and length of experience with patients with LTCs on practicing Clinical Self Management Support, Patient Centeredness and Organizational Self Management Support.

The Patient Centeredness was the most frequently endorsed scale by the sample, and suggests that clinicians see a key role in customizing the care provided by them to the specific needs and lifestyle of the individual, such that there was a ceiling effect in this sample. None of the variables we identified predicted practicing Patient Centeredness, as there was very little variance in the range of responses.

The second most often practiced activities were those of the Clinical Self Management Support scale, which relate more specifically to lifestyle and behaviour change techniques. Over $80 \%$ of respondents reported ensuring that the patient is comfortable with a jointly agreed management plan and decisions, and sharing power and responsibility with the patient. This is important as it has been shown that patients engage in self management only if they play an active role in developing their self care plan and if proposed solutions are coherent with patients' beliefs and values [20-21]. However, fewer clinicians endorsed the practice of joint agenda setting prior to the consultation. The lack of these may result in covering only issues important for the clinician but not necessarily for the patient. Not being able to discuss important treatment issues and resolve the patient's concerns may result in lower adherence to treatment recommendations and poorer health outcomes [22]. We also found that less than $40 \%$ of respondents discussed with the patient joining condition related support groups, which is an important practice because there is evidence that self management support programmes can have a positive impact on clinical [23] and psychosocial variables [24].

Clinicians who spend over $50 \%$ of their working week with patients with LTCs and who had recently attended professional training in self management support are more likely to engage in Clinical Self Management Support. Attending professional training helps clinicians to develop relevant skills, however the fact that clinicians who spend more time in direct contact with patients with LTC are more likely to engage in this area of self management support may mean that not only acquiring skills, but also having an opportunity to regularly practice them is crucial for maintaining new behaviours. This assumption is supported by the results of multiple regression showing that combined training and intensity of experience with LTC are the most important predicators of practicing self management support.

The results for the sub scale Organization of Services to Support Self Management showed that these practices are much less commonly part of routine care, yet they are often the focus of service quality improvement programmes. Only around a half of participants jointly agreed with patient how the progress will be followed up, which means for those who do not prioritise this in their practice, their patients may experience problems but be unclear as to how or when or from whom to seek 
healthcare system support. This may result in low compliance [22]. Less than $50 \%$ of clinicians were engaged in building their patient care team with other professionals, which may simply mean their work is carried out as individuals, and their patients are not able to access the full range of skilled support. Only about a quarter of participants routinely had self management records included in the patient's documentation. In that case patients' plans and achievements may not available at later consultations for others to use, which prevents regular and effective monitoring of the patient's progress. Only a quarter (26\%) gave patients a choice about the care team member who will coordinate their care plan. Having limited patient choice may reflect the lack of team working noted above, as well as the fact that the care team coordination is already prescribed - e.g. to a specialist nurse.

Unsurprisingly, clinicians with largely managerial or academic roles were more likely to undertake Organizational Self Management Support. Nurses and doctors were significantly more likely to engage in Organizational Self Management Support in comparison to professionals allied to medicine, which may suggest gaps in professional training, lack of exposure to quality improvement methods in their training, or lack of seniority. The strongest predicators of engaging in Organizational Self Management support were attending professional training and the intensity of experience with patients with LTCs which again may suggest that not only theoretical knowledge, but also having an opportunity to regularly apply newly acquired skills are important factors in supporting self management.

There several important limitations to this research. First of all the size of the sample is not big enough to guarantee its representativeness. The response rate was only $13 \%$. We may assume that clinicians who responded were those with particular interest in self management support and thus were more skilled in comparison to their colleagues who have no particular interest in this area. Regression analysis was conducted on 122 eligible case with four potential predicators. It is recommended that the number of cases should be 10-20 times larger than the number of predictors and we met this criterion, but the results should be retested to assess the stability of the results. The PSMS questionnaire we used is a self assessment survey that is open to many self presentational and social desirability biases. We attempted to minimize the impact of these factors by assuring respondents' anonymity.

The study has shown that the most important factors associated with practicing self management support for patients with LTCS are intensity of experience with patients with LTCs and having recently attended professional training addressing the principles and practice of self management support. We found that clinicians who recently attended professional training and spend more time at work on direct care/contact with patients with LTC are more likely to engage in Clinical and Organizational Self Management Support. The interaction of training and intensive work with patients with LTC seems to have the most powerful effect on undertaking self management support in those two areas. These findings suggest that managers should concentrate resources on those who have not yet had relevant training, irrespective of the intensity of working contact with patients with LTCs. It seems logical, given the association between these variables and the somewhat infrequent use of Organisational Self Management skills, to target training in quality improvement work in health care on these whose training has not yet prepared them for this, and whose work provides high intensity of contact with people with LTCS. This would also prepare the ground for the Government's goal to be achieved by 2010, to offer every patient with long term condition a personalized care plan [2] and support self management between appointments.

We conclude that to facilitate clinicians' practice of self management support for patients with LTC it is important to provide relevant professional training increasing clinicians' feeling of competence in relation to self management support. Perhaps apart from covering theoretical aspects of self management support and teaching particular techniques, the training should also cover behaviour and lifestyle change and organisational skills to support self management. This may include familiarising clinicians with rules of setting achievable goals, coaching patients to achieve their goals, developing problem solving skills, and if necessary knowing who to refer patients to so they can obtain necessary support in changing they health behaviours. Also, disseminating organisational policy documents on long term conditions management and self management support as well as making clinicians aware how they can influence this policy would be a great benefit. Perhaps organising regular clinical teams' meetings to discuss self management support policy and testing and implementing potential changes would help achieve this goal. Basing on our finding we also assume that building patient care teams with professionals having complimentary skills may increase undertaking self management support by its members. Having a team dedicating much of its time to caring for patients with particular LTCS, attending the same professional training and educational events and having regular meetings to discuss issues arising 
around continuity of care, organization of services provided, self management support as well as individual patients' care plans would potentially increase each clinicians' competence level as well as build up a sense of being supported by other team members and hence increase the frequency and effectiveness of self management provided to patients with LTCS.

\section{References}

1. Epping-Jordan JE, Pruitt SD, Bengoa R, Wagner EH. Improving the quality of health care for chronic conditions. Qual Saf Health Care 2005; 13: 299-305.

2. Department of Health (2007) Supporting People with Long Term Conditions. http://www.dh.gov.uk/en/Publicationsandstatistics/Publications/PublicationsPolicyAndGuidance /Browsable/DH_4100317

3. Hibbard JH, Collins A, Baker L. Clinician activation: physician beliefs about patient self-management. Background paper for The Commonwealth Fund and The Nuffield Trust; $9^{\text {th }}$ International Meeting on Quality of Health Care 2008.

4. Bandura A. Self-efficacy: toward a unifying theory of behavioral change. Psychol Rev 1977; 84: 191-215.

5. Janz NK, Becker MH. The health belief model: a decade later. Health Educ Q 1984; 11: 1-47.

6. Rollnick S, Kinnersley P, Stott N. Methods of helping patients with behaviour change. Br Med J 1993; 307: 188-190.

7. Prochaska JO, Velicer WF. The transtheoretical model of health behavior change. Am J Health Promot 1997; 12: 38-48.

8. Coulter A. Partnerships with patients: the pros and cons of shared clinical decision-making. J Health Serv Res Policy 1997; 2: 112-21.

9. Clark NM, Gong M. Management of chronic disease by practitioners and patients: are we teaching the wrong things? Br Med J 2000; 320: 572-5.

10. Holman HR, Lorig, K. Patients as partners in managing chronic disease. Br Med J 2000; 320: 526-7.

11. Stewart M, Brown JB, Boon H, Galajda J, Meredith L, Sangster M. Evidence on patient-doctor communication. Cancer Prev Control 1999; 3: 25-30.

12. Stewart M, Brown JB, Donner A, et al. The impact of patient-centered care on patient outcomes. J Fam Pract 2000; 49: 796-804.

13. Epstein RM. The science of patient centered care. Commentary. J Fam Pract 2000; 49: 805-7.

14. Corben S, Rosen R. Self-management for long-term conditions: patients' perspectives on the way ahead. 2005. www.kingsfund.org.uk

15. Newman S. Engaging patients in managing their cardiovascular health. Heart 2004, 90:, iv9-iv13.

16. Katon $M$, Von Korff $M$, Lin M, Simon G. Rethinking practitioner roles in chronic illness: the specialist, primary care physician, and the practice nurse. Gen Hosp Psychiatry 2001; 3: 138-44.

17. Rothman AA, Wagner EH. Chronic Illness Management: What Is the Role of Primary Care? Ann Intern Med 2003; 138: 256-61.

18. Wilson T, Buck D, Ham C. Rising to the challenge: will the NHS support people with long term conditions? $\mathrm{Br}$ Med J 2005; 330: 657-61.

19. Kosmala-Anderson J, Wallace LM, Turner A. Development and Psychometric Properties of a Self Report Measure to
Assess Clinicians' Practices in Self Management Support for Patients with Long Term Conditions. Patient Education and Counseling, 2010, in press.

20. DiMatteo MR Enhancing patient adherence to medical recommendations. JAMA 1994; 271: 79-83

21. World Health Organization Adherence to long term therapies: evidence for action. 2003.

22. Vermeire E, Hearnshaw H, Van Royen P, Denekens J. Patient adherence to treatment: three decades of researcher. A comprehensive review. J Clin Pharm Ther 2001; 26: 331-42.

23. Chodosh, J, Morton, SC, Mojica, et al. Meta-Analysis: Chronic Disease Self-Management Programs for Older Adults. Ann Intern Med 2005; 143: 427-36.

24. Foster G, Taylor SJC, Eldridge SE, Ramsay J, Griffiths CJ. Self management education programmes by lay leaders for people with chronic conditions. The Cochrane Database of Systematic Reviews, issue 4. John Wiley \& Sons, Ltd, 2007. 